\title{
A REAL-TIME AND NON INTRUSIVE STRUCTURAL HEALTH MONITORING SYSTEM FOR BUILDINGS
}

\author{
Berardo Naticchia, Alberto Giretti, Alessandro Carbonari, Massimo Vaccarini \\ Università Politecnica delle Marche, DACS Department - Division of Building Construction, \\ Ancona, Italy \\ b.naticchia@univpm.it, a.giretti@univpm.it, alessandro.carbonari@univpm.it, \\ m.vaccarini@univpm.it
}

\begin{abstract}
The constant innovation in the development of sensing devices for detecting health status of buildings, highlights the importance of this professional field. In fact, correct diagnosis would prevent many pathologies, driving maintenance interventions towards timely and well targeted operations. This paper describes the development of a monitoring system prototype, conceived to be very low intrusive, that can be applied also on existing buildings, without any need to predispose chases for power or communication cables. Its adoption is expected to be useful not only for standard health status monitoring of buildings during their lifecycle, but also for automated monitoring of old buildings during the conduction of works for their renovation. As a matter of fact in this case accidents due to unexpected collapses during work execution are very common, hence the static condition of buildings should be continuously checked automatically and alerts sent in case of high risk of failures. The sensors under development have been programmed in such a way to be able to monitor rotations and send alerts in case critical thresholds are overcome.
\end{abstract}

KEYWORDS: structural health monitoring, construction refurbishment, health and safety.

\section{INTRODUCTION}

Structural stability is the most fundamental requirement of buildings. Structural monitoring is aimed at assessing the condition of buildings' structure on the basis of the their history and on measurements, carried out by means of sensor devices placed at the structural critical points. Recorded data are then used to qualify the current status of the structure, pointing out the eventual growing of pathologies through the observation of critical symptoms like fractures, significant displacements and tilt. The set-up of monitoring networks usually requires careful design and a considerable installation effort because of cabling and tunings. This makes the embedding of monitoring sensor networks affordable on new buildings, but rather complex and invasive on existing ones. This aspect becomes more critical in the case of building rehabilitation, where structural monitoring is used for safety purpose, predicting the eventual occurrence of collapses. In construction sites it is well known that cable overlays may have significant impact on the required flexibility of the worksite layout, and are therefore avoided as much as possible.

In this paper we exploit new technologies providing wireless sensor networks, with modular structure and short duty cycles, which are used for transmission of data, recorded by low cost and low invasive sensors deployed throughout buildings. In particular, we will report about a 
prototype of a structural monitoring system which combines accurate and reliable tilt sensors with a low power zig-bee based data transmission network, that can be easily installed on any kind of building and working environments. The system can be managed and reconfigured remotely and used for both building life-cycle monitoring and safety improvement during renovation interventions of existing buildings.

\section{STATE OF THE ART AND APPLICATION DOMAINS}

Structural health monitoring is being applied for a lot of tasks, among them for monitoring long-term structural degradation or deterioration, moreover in case the structure underwent an extreme loading event (Brownjohn, 2007). The need for this practice is evident when thinking about the dangerous consequences that could follow from the use of structurally deficient or functionally obsolete buildings, moreover those ones located in seismic areas or subject to other types of extreme events.

Many approaches have been developed over the years, most of them focusing on some statistic methodologies to detect data from measured response, collected by sensor devices (Sohn, 2007). However, measuring is a difficult task for a number of reasons, among them we cite measurement noise, inadequate number or accuracy of sensors, scarce separation of environmental factors. Due to the considerable influence of noisy factors, the development of reliable systems has always been a tough challenge. Thus far, full-scale structural health monitoring installations have been based on traditional wired based systems, whose reliability have been widely tested over the past decades and they have been proved to be applicable to a number of differing situations, with changing objectives, outcomes and application domains. Given the complex operations involved by those wired-based installations, they have been integrated moreover in new constructions. Some representative examples are given by installation on high towers, on retrofitted buildings and on bridges. (Ni et al., 2009) have developed a six module structural health monitoring system, and installed it on a super-tall tower with a height of $610 \mathrm{~m}$. The system acquires data from sensors, performs structural health evaluation and integrates a wide range of sensors, relative to loading, structural responses and environmental parameters monitoring. They all are wired connected to a central acquisition system located in the building itself. Accelerometers, gages, fibre-optic strain sensors, laser based displacement sensors, and acoustic emission sensors and so on, have also been used to measure the changing characteristics of a building under retrofitting, as the construction progressed (Chassiakos et al., 2007). Very widespread is also remote monitoring of bridge structures (Mehrani et al., 2009), as their deterioration must be estimated and life time expectancy predicted, in order to assure user safety, which is critical also in many other public civil works (Bhalla et al., 2005).

However, the cost of adequately equipping a large structure with a wired-based monitoring system has been shown to be very high, not only for the number of sensor devices, but moreover for installation costs (Lynch and Loh, 2006) (Kurata et al., 2006). For that reason, engineers and researchers are seeking for new wireless sensor systems, that would render it cheaper and would favour its spread in the market, with clear consequent social benefits. This new requirement imposes a set of new challenges also in the choice of adequate sensors, to be integrated within the monitoring systems, as they must be low-voltage powered and low consumption type, they must guarantee high accuracy with low communication data rate and other important constraints that will be highlighted in the next paragraph. 
Finally, the development of such a wireless sensor system would allow another interesting application for health and safety management in retrofitting of old buildings (Figure 1-a): as operating on deficient buildings is dangerous for their possibility of collapsing, then a real time structural health monitoring system installed on the structure interested by retrofitting, would be useful to timely detect possible upcoming damages. It would advise to evacuate before damages would turn into collapses. An hypothesis of network topology to this aim has been sketched in Figure 1-b and will be further detailed in the following of this paper.

a)

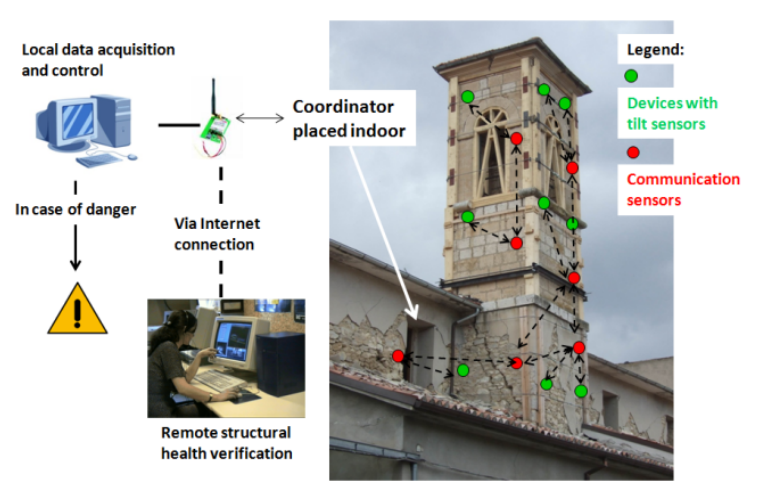

b)

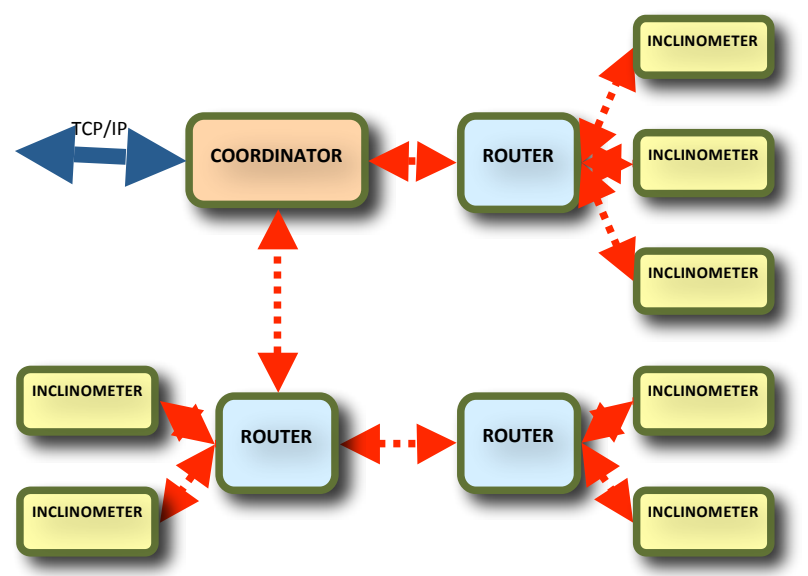

Figure 1: Concept of wireless real time monitoring in a construction site (a) and network topology (b).

\section{REQUIREMENT ANALYSIS}

As stated in the previous paragraph, the most challenging application foreseen for the system regards its adoption in large construction sites for protecting workers involved in building refurbishment. In this case, the system must comply with two sets of requirements: to not interfere with operations in progress and to reliably monitor structural behaviour and alert in case of danger.

As far as concerns the first set of requirements, the system has been developed as a set of small, light and easily deployable devices, which wirelessly communicate one another and to a central server responsible for data acquisition. Two kind of deployable devices have been developed (Figure 1-b): the first kind having the main task of monitoring the structural behaviour of unsafe structures and broadcast these data (e.g. through an inclinometer); the second kind having the main task of forwarding those data towards the Central Application (i.e. routers). All those devices are expected to work outdoor, hence they must even be resistant to harsh environmental conditions.

As far as concerns the second set of requirements, several strains can be used for assessing structural health of buildings, like displacements and rotations. In this first research step, it was decided to develop a prototype of sensor equipped with an inclinometer, as this kind of measure was considered to be the simplest to be performed; for example it does not need a fixed reference point, like for displacement measures. Instead tilt measures are all relative and expressed as variations with respect to an initial value, fixed during the installation and calibration phase. Selection of the type of tilt sensor to be included in the system has been made on the basis of a preliminary estimation of the expected rotations in such buildings. The masonry tower depicted in Figure 2-a was taken as a test-case. The purpose of tilt measures is 
mainly the one of providing an evaluation base for sending an alert as soon as the structure is subject to a strain that could be dangerous for its users' safety.

Hence, a structural survey according to current legislation (Eurocode 8, 2004) regarding the structure's response under seismic actions was conducted. Eurocode 8 defines two kinds of limit states: ultimate limit states (associated with collapse or other form of structural failure) and damage limitations states (associated with damages, beyond which specified service requirements are no longer met). As the monitoring system is expected to alert before the structure reaches collapse, then deformations corresponding to damage limitation states have been estimated. The earthquake motion at a given point has been represented as an "elastic response spectrum", whose shape was computed for the particular location of the building, set in an Italian town (Senigallia, Ancona). Then a finite element linear elastic model of the structure has been built and simulated through SAP $2000^{\mathrm{TM}}$ software program (Figure 2-b). The main parameters characterizing the masonry structure are: density equals to $18 \mathrm{kN} / \mathrm{m}^{3}$, Young modulus equals to $5 \cdot 10^{6} \mathrm{kN} / \mathrm{m}^{2}$, maximum axial compression of $5300 \mathrm{kN} / \mathrm{m}^{2}$ and maximum shear resistance equals to $200 \mathrm{kN} / \mathrm{m}^{2}$. It came out that in the point where the maximum rotation has been recorded, marked through a red circle in Figure 2-c, a tilt value of $0.15^{\circ}$ has been detected. Hence the system is expected to have a repeatability of at least $0.05^{\circ}$ or better, in order to be able to advice in advance, before reaching the thresholds of damage limitation state, beyond which damages and dangerous instability for users (or workers in case of renovation) would be experienced. The system development phase has been conducted to produce a sensor complying with such requirements.

(a)

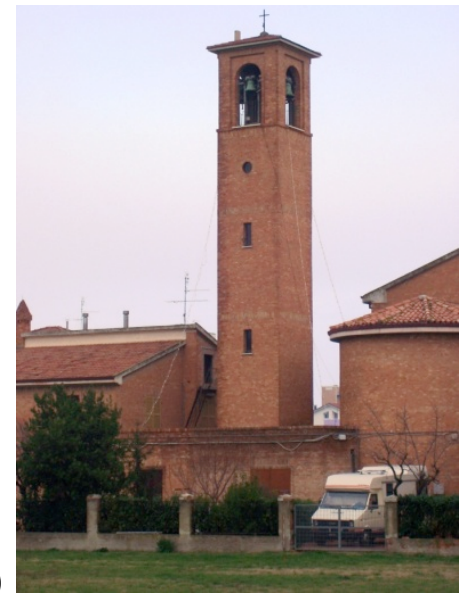

(b)

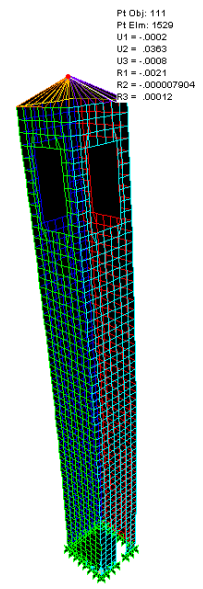

(c)

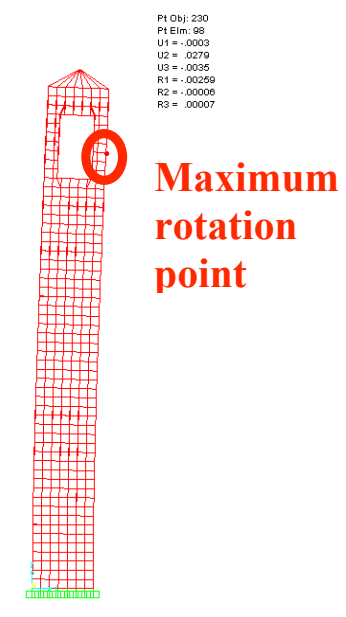

Figure 2: Masonry tower taken as a test case for tilt rotation's estimation (a); its finite element model (b) and lateral view of the tower subject to maximum deformations (c).

\section{SYSTEM DEVELOPMENT}

\section{System overview and components}

As the system must be easily deployable throughout construction sites and expandable according to work progress, then it was designed as a wireless and untethered set up. Figure 1-b depicts a feasible solution for its whole architecture, which was made up of the following components: one or more coordinators used to initiate network formation, acting as 802.15.4 PAN coordinator (FFD) and performing applications; fixed devices (or routers) installed at known locations and having the task to perform multi-hop routing of messages; end devices 
equipped with inclinometers and installed at critical points of the structure, which do not participate in routing but are used to acquire inclination measures and send them to the coordinator which, in turn, transfer it to the Application Server, in charge of checking building's health status. The communication system was developed in collaboration with the Italian Company Smart Space Solutions srl, which designed it as a set of hardware and software components based on the IEEE 802.15.4 standard medium access and Zigbee stack communication protocol. It owns also very low power features, which are assured thanks to a reduced router's duty cycle in asynchronous mode, and provides a power autonomy of about 2-3 years. More details are given in the paper (Carbonari et al., 2010).

A device performing critical tasks in the system was obviously the node equipped with a tilt sensor, which is in charge of continuously monitoring structure inclinations and conveying those values to the central system for stability checking. In addition, as several nodes of this type are expected to be installed on the structure and to be held there for one or more years, then the node should be easily installable, fast to calibrate and resistant to harsh environmental conditions.

\section{Development of the sensor node}

The external look of the one end device equipped with a tilt sensor is shown in Figure 3-a and detailed drawings in Figure 3-b: all the electronic equipment, including tilt sensor and communication system, is contained in a water tight plastic box (produced by Gewiss ${ }^{\mathrm{TM}}$ ), which assures resistance to outdoor environments and do not interfere with wave propagation; the plastic packaging is fixed on an adjustable flange, fixed to a metal plate through the use of three screws (one at the top and two at the bottom) for setting the sensor on a vertical position and allowing it to start measuring from the middle of its operating range, hence exploiting its total range; finally, the metal plate can be fixed in several ways to various kinds of structural surfaces, from reinforced concrete elements to masonry walls.

A True Tilt $^{\mathrm{TM}}$ sensor, patented and produced by the Fredericks Company ${ }^{\mathrm{TM}}$, has been embedded in the system. It is an electrolytic inclination sensor with a maximum operating range of $\pm 2^{\circ}$ and, thanks to its high resistive behaviour; it does not sensibly affect power consumption of the whole device. Its resolution is better than 1 arc second (about $0.0003^{\circ}$ ) and the null repeatability is better than 5 arc seconds, equals to $0.0014^{\circ}$. A schematic of the electronics implemented in the inclinometer is shown in Figure 3-c. Since the tilt sensor must be driven with a zero mean value supply signal applied between the two ends (pin 1 and 3), a decoupling capacitor has been introduced between the digital buffer and the tilt sensor. Its value must be big enough to limit the voltage decay during analog to digital (AD) conversion interval and to allow, at the same time, complete discharge of the capacitor during turn-off period: otherwise, the stability of the sensor reading would be compromised and the sensitivity would be sensitively reduced (Figure 3-d). In order to save power, the microcontroller $(\mu \mathrm{C})$ wakes-up every second, acquires data and transmits it only in case a significant variation has been detected or if a defined timeout has been reached. The sequence implemented at each step by the $\mu \mathrm{C}$ is the following:

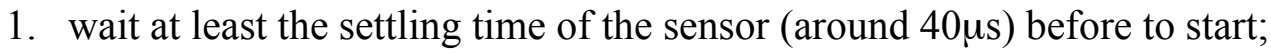

2. sample and hold the analog voltage;

3. perform the acquisition;

4. turn off the sensor;

5. evaluate acquired data; 
6. if a significant variation of the reading is detected, transmit all data acquired up to now, otherwise store the last value;

7. return to sleep mode.

a)

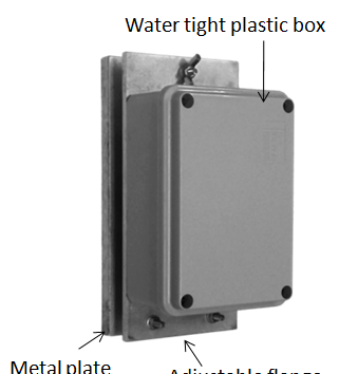

b)

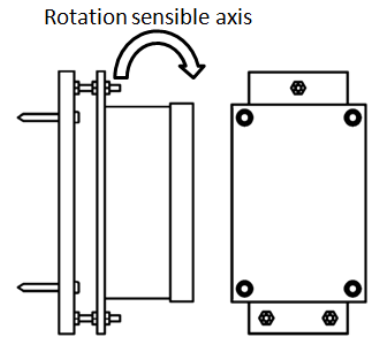

c)

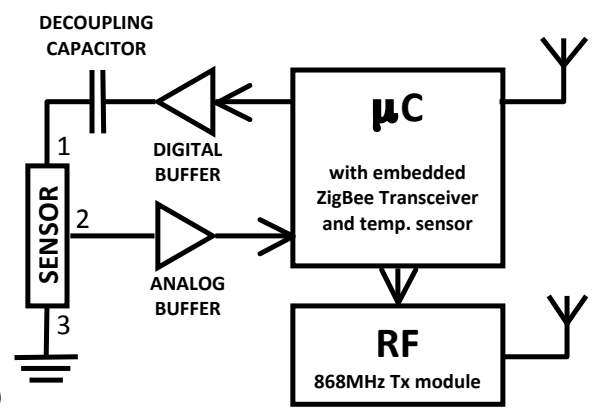

d)

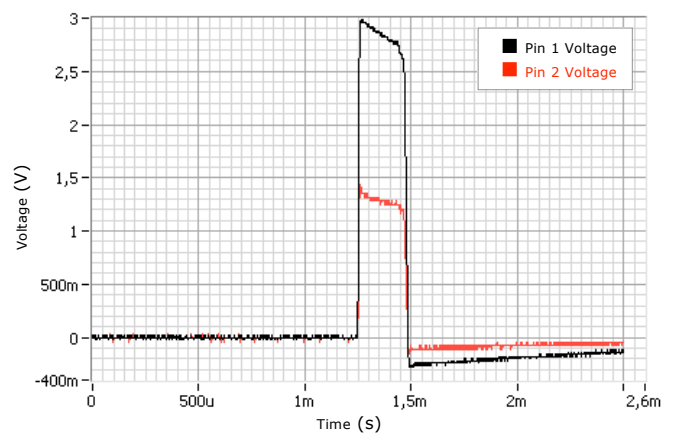

Figure 3: Inclinometer sensor node (a); its front and lateral views (b), scheme of the electronic acquisition and communication system (c), sensitivity estimations (d).

The acquired and transmitted value is an integer number corresponding to the AD conversion of the input voltage. This raw data is server side, further elaborated in order to convert it in inclination angle using a predefined calibration curve. A calibration test has been preliminarily performed to find out the mathematical relationship linking the readings from the True Tilt ${ }^{\mathrm{TM}}$ sensor and the actual inclinations measured. As shown in Figure 4-a, the inclinometer under calibration was positioned on an adjustable inclined plan, through the use of a screw on one end, while the other end was left free to rotate around a hinge. The inclination of this plan was varied within the operating range of the inclinometer, and its readings were compared with the ones collected by a mechanical inclinometer produced by OfficineGalileo $^{\mathrm{TM}}$ (used as a benchmark) and based on the spirit level principle. It has a very accurate resolution of 1 arc second and an operating range of $\pm 1^{\circ}$.

Figure 4-b shows the relationship inferred from the calibration process. It can be noticed that the range has been limited between about $-0.3^{\circ}$ and $+0.3^{\circ}$ for two main reasons: out of this range the inclinometer loses its almost linear range; in addition, preliminary estimation of the rotations to be measured in buildings showed that $\pm 0.3^{\circ}$ interval is enough wide for its application domain. The mathematical relationship printed on the diagram in Figure 4-b was estimated as a third order polynomial relationship, as it gave back the best fitting, with respect to the 34 measured values during calibration. It gives back a very high $\mathrm{R}^{2}$ parameter, equals to 0.9997, whose top (and best) index value is equals to 1 (Brockwell and Davis, 1991). The readings acquired by the wireless acquisition system were limited between 700 and 1340, that are plotted according to a 1:1000 scale. This relationship was then implemented in the acquisition system to convert electric readings into actual inclinations. 
a)

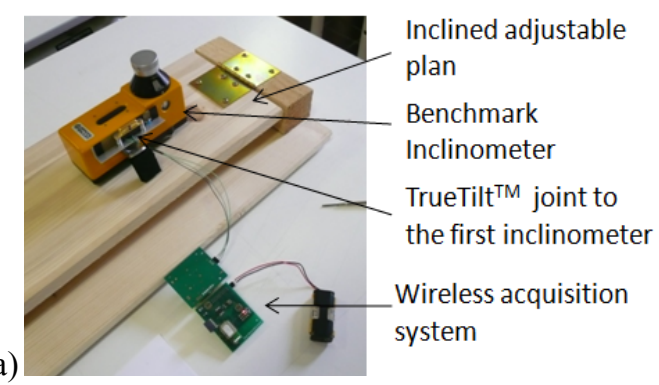

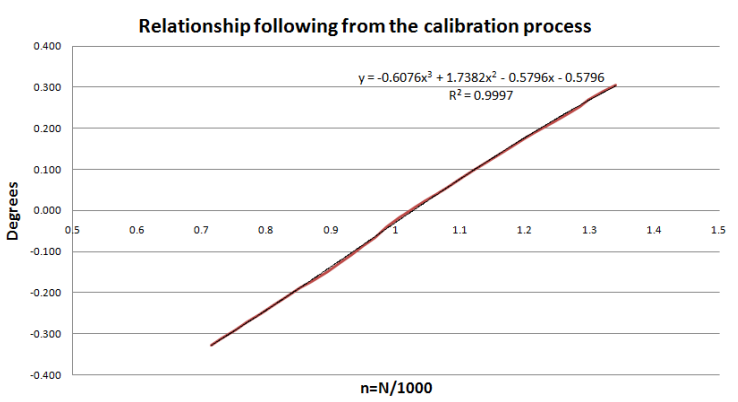

Figure 4: calibration process (a) and relationship between readings from inclinometer and actual inclination (b).

Among the features of the inclinometer used, there is also sensitivity to temperature variations. Its producer provides a temperature coefficient of $\pm 0.75 \operatorname{arcsecond} /{ }^{\circ} \mathrm{C}$, which will affect outdoor measures as a consequence. For that reason, several laboratory tests have been performed to estimate how much such a variations would influence the readings acquired by the inclinometer. It is thought that the readings will be filtered according to the temperature course. Temperature is monitored through the use of a sensor embedded in the $\mu \mathrm{C}$ that is enclosed in the watertight box together with the tilt sensor. The accuracy, as declared by the manufacturer, is $\pm 2^{\circ} \mathrm{C}$ in the range $\left[-20^{\circ} \mathrm{C},+80^{\circ} \mathrm{C}\right]$, after a one point linear calibration has been performed.

To the aim of estimating sensor readings errors due to temperature variation, two prototypes of tilt sensor were exposed to high and rapidly varying temperature, generated by an infrared lamp, positioned at a distance of $0.6 \mathrm{~m}$ from the wall the inclinometers had been installed on (Figure 5-a). The inclination plots reported in Figure 5-b show that tilt errors determined by very high and sudden temperature risings are lower than $0.016^{\circ}$ for the first tilt sensor and lower than $0.033^{\circ}$ for the second sensors. The difference can be due to the different impact of infrared rays from the lamp on the sensors and to the intrinsic different features of the inclinometers and of their acquisition systems. The reading peaks were obtained bringing the temperature from the environmental value of $15-16^{\circ} \mathrm{C}$ up to $41^{\circ} \mathrm{C}$ and $46^{\circ} \mathrm{C}$ for the first (red line) and second (blue line) tilt sensors respectively, within a time window of only 2 hours. To be noticed that such temperatures can be reached outdoor only in case the sensors are very intensely irradiated by the sun. The rate of temperature increase simulated in this laboratory trial could be hardly repeated in real conditions, hence it represents the extreme case that could reasonably happen during real installations on buildings. It is worth noting that the maximum tilt variation recorded, equals to $0.033^{\circ}$, is well lower than the accuracy threshold required for structural monitoring of $0.05^{\circ}$, deriving from our requirement analysis.

Finally another test with a twofold aim was performed: sensor resistance to harsh conditions and communication range. The first was aimed at verifying its suitability for working outdoor, where temperatures could realistically vary between -20 and $+50^{\circ} \mathrm{C}$. The second was aimed at estimating the maximum distance these sensors can be placed from each other, which is strongly related with installation costs and time efforts. Both nodes equipped with tilt sensors and communication sensors were driven to $-20^{\circ} \mathrm{C}$, by means of the climatic chamber type "Challenge 250" depicted in Fig. 6-a, which covers a range of temperatures from $-40^{\circ} \mathrm{C}$ to $180^{\circ} \mathrm{C}$. 
a)

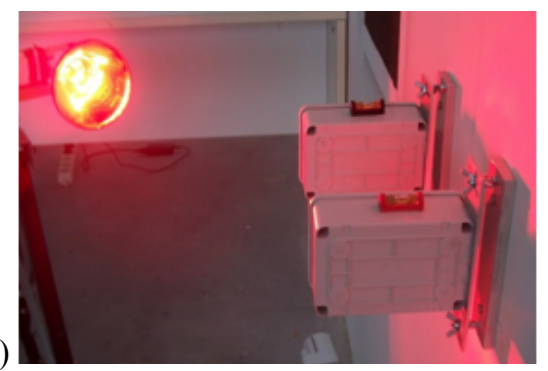

b)

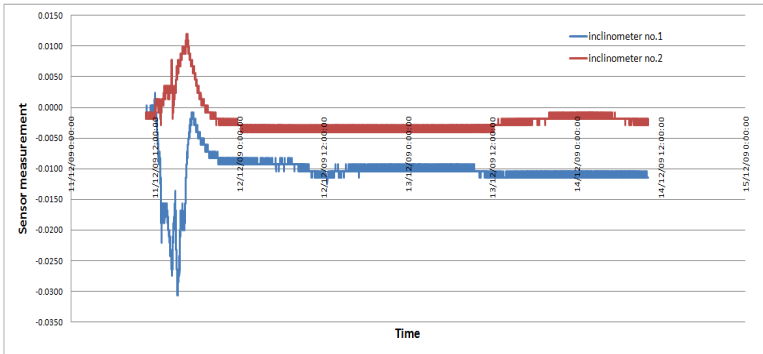

Figure 5: prototypes of tilt nodes exposed to irradiating lamps (a) and inclination errors determined by suddenly increasing temperature (b).

The temperature cycle was built in order to bring sensors' temperature from environmental value to $-20^{\circ} \mathrm{C}$ within $45 \mathrm{~min}$, then it was kept constant for $1 \mathrm{~h}$, in order to allow the whole system reaching thermal equilibrium. After that time window, the nodes were extracted and their communication ranges measured through the configuration shown in Figures 6-b and 6c: the end device was put very close to three routers, which then communicated with router no. 1, close to the coordinator, in charge of data collection. At every measure, the signal quality of the three routers was measured, in order to infer the most suitable protection against extreme temperature values and the moisture. Each of the three routers was protected in a different way: router no. 2 was not protected, but only inserted inside a water tight box containing hygroscopic salts; router no. 557 was covered by a thin silicone conformal coating, specifically designed for the protection of electronic circuit in the automotive sector, with a dielectric strength of $90 \mathrm{kV} / \mathrm{mm}$ and dielectric constant of 3.95 at $1 \mathrm{MHz}$; router no. aac was covered with a red coloured silicone conformal coating, having the same dielectric properties of the previous one, but with a higher density and coloured using a copper powder made pigment.

Then the quality of signal received by router no. 1 from the three routers was estimated from several distances, varying between 5 and $40 \mathrm{~m}$, as the average percentage of packets received, with respect to all the packets sent (one every $5 \mathrm{~s}$ ). While varying their positions, routers no. 2 and 557 gave back reliable results until a distance of $40 \mathrm{~m}$, having a high mean quality of signal equal to $80 \%$ and $85 \%$ respectively. Instead, router no. aac behaves generally bad, with an average quality of signal equals to $45 \%$.

a)

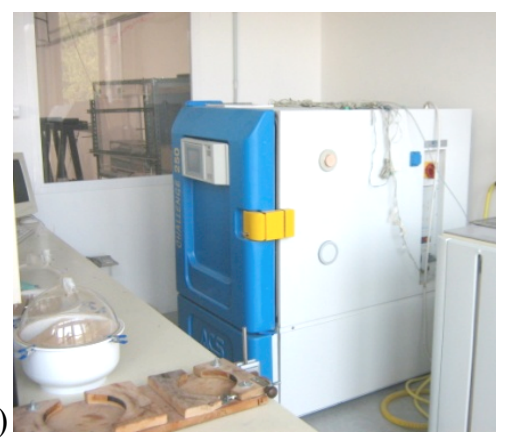

b)

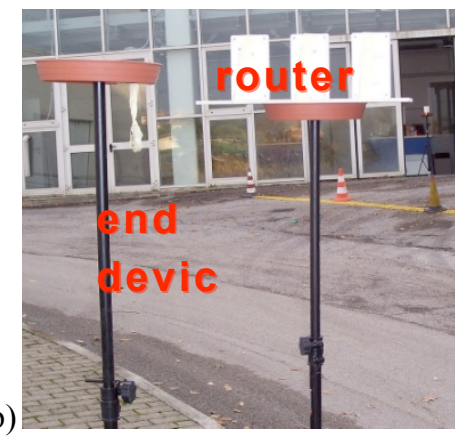

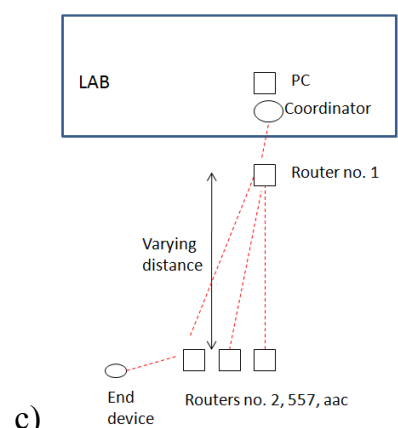

c) communication range among the devices $(b, c)$.

\section{Estimation of power consumption}

Power consumption of the inclinometers is a key issue for their autonomy and non-wired installation. Figure 7 and Table 1 show the measured current consumption of the devices when powered with a $3 V$ battery pack and for two different conditions: Figure 7-a is relative 
to the acquisition followed by the transmission, whereas Figure 7-b is relative to the condition in which after acquisition no significant variation have been detected by the sensor. In addition, a power consumption of $0,04 \mathrm{~mA}$ should be added to all the time for which the device is in sleep mode. With these consumptions, the estimated lifetime of an inclinometer strongly depends on the frequency of transmissions and on the sampling interval. By considering a sampling interval of 1 second and a lithium battery pack with capacity of $3000 \mathrm{mAh}$, the resulting estimated autonomy varies from about 6 to 44 months when transmission interval varies from 1 to 25 seconds respectively. Since in a normal functioning mode the transmission is done only when an anomalous variation is detected or the maximum length of the buffer (i.e. 25) is reached, then the usual device autonomy is more than 3 years.

Table 1: Current consumption estimations

\begin{tabular}{lcc}
\hline Case & Duration & Mean Current \\
\hline Acq. + Tx & $29 \mathrm{~ms}$ & $20 \mathrm{~mA}$ \\
\hline Acq. & $2,6 \mathrm{~ms}$ & $12 \mathrm{~mA}$ \\
\hline SLEEP & $1 \mathrm{~s}$ & $0,04 \mathrm{~mA}$ \\
\hline
\end{tabular}
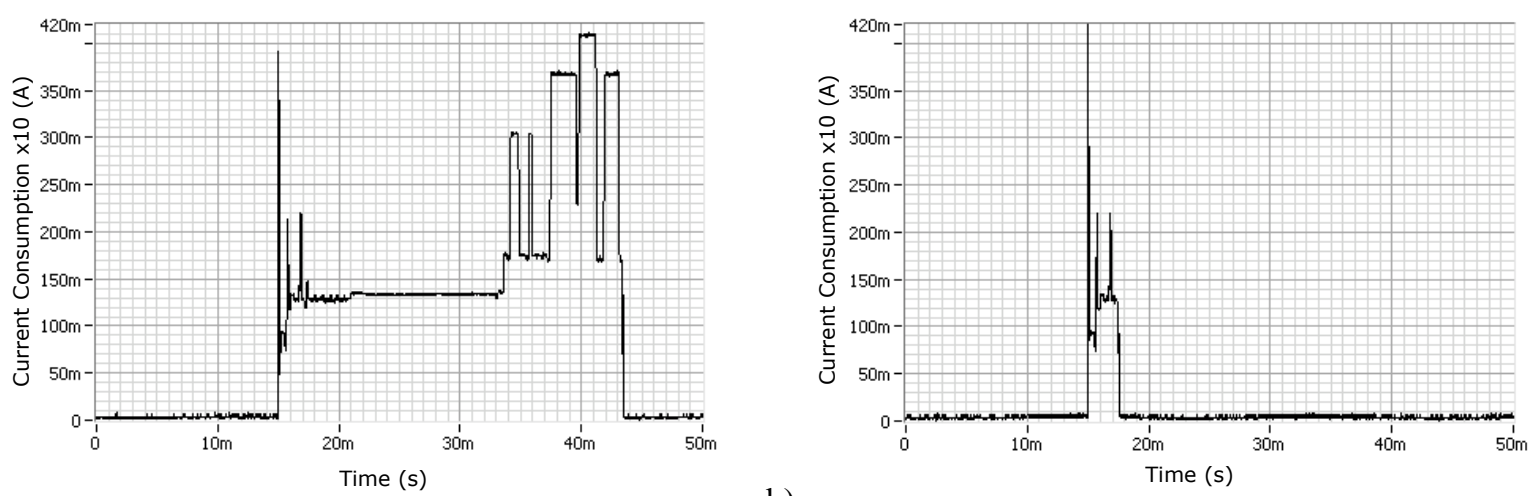

a)

b)

Figure 7: Current consumption of the inclinometer with (a) and without (b) data transmission.

\section{CONCLUSION}

Given the wide testing carried on, it is possible to assume a preliminary control logic, to be implemented at the Central Application level, according to the data recorded from the sensor system. A starting calibration must be performed at the beginning, and then make an iterative control on the structure behaviour. The thresholds can be computed according to what performed for the building in Figure 2-a, through a structural analysis on buildings, but the orders of magnitude found out for our test case are expected have a general validity.

Sensors are first installed and adjusted upright; the values acquired at this stage are set as reference points. Then, readings will be collected by each sensor and compared with two thresholds: the lower corresponding to damage limitations state and the higher corresponding to ultimate limit states. In case the lower is overcome, then a check by structural engineers on building's health status will be required; in case the higher level is overcome, then a red alarm must be sent, as the building needs rapid evacuation. In case none of them are reached, then another iteration will be performed.

Finally, the monitoring system developed in this paper for controlling health status of buildings was shown to be suitable for installation outdoor, and to perform continuous 
checking of masonry structures evolution. Its main advantages are: low invasiveness; easy installation; high frequency data acquisition and real time control of health status; resistance to harsh conditions. Given the good results deriving from laboratory surveys, further testing on real structures will be executed and its long term behaviour will be evaluated.

\section{REFERENCES}

Brownjohn, J. M. W. (2007). Structural health monitoring of civil infrastructure. Philosophical Transactions of the Royal Society A: Mathematical, Physical and Engineering Sciences, 365, 589-622.

Sohn, H. (2007). Effects of environmental and operational variability on structural health monitoring. Philosophical Transactions of the Royal Society A: Mathematical, Physical and Engineering Sciences, 365(1851), 539-560.

Kurata, N., Saruwatari, S. and Morikawa, H. (2006) Ubiquitous Structural Monitoring using wireless Sensor Networks, IEEE International Symposium on Intelligent Signal Processing and Communication Systems (ISPACS 2006), Yonago-Japan.

Lynch, J. P., and Loh, K. J. (2006). A summary review of wireless sensors and sensor networks for structural health monitoring. The Shock and Vibration Digest, 38(2).

Y. Q. Ni, Y. Xia, W. Y. Liao and J. M. Ko (2009) Technology innovation in developing the structural health monitoring system for Guangzhou New TV Tower, Structural Control And Health Monitoring, 16, 73-98.

A. G. Chassiakos, S. F. Masri, R. D. Nayeri, J. P. Caffrey, G. Tzong and H. P. Chen (2007) Use of vibration monitoring data to track structural changes in a retrofitted building, Structural Control And Health Monitoring, 14, 218-238.

Ebrahim Mehrani, Ashraf Ayoub, Amir Ayou (2009) Evaluation of fiber optic sensors for remote health monitoring of bridge structures, Materials and Structures, 42, 183-199.

S. Bhalla, Y.W. Yang, J. Zhao, C.K. Soh, (2005) Structural health monitoring of underground facilities - Technological issues and challenges, Tunnelling and Underground Space Technology, 20, 487-500.

Eurocode 8 (2004) Design of structures for earthquake resistance - Part 1: General rules, seismic actions and rules for buildings.

Alessandro Carbonari, Angelo Biscotti, Berardo Naticchia, Federico Robuffo, Mario De Grassi (2010) A management system against major risk accidents in large construction sites, forthcoming in Proceedings of the 27th International Symposium on Automation and Robotics in Construction (ISARC 2010), June 25-27, Bratislava, Slovakia.

Brockwell, P. J. e Davis, R. A. (1991). Time Series: Theory and Methods. Springer-Verlag, 2nd ed. 